
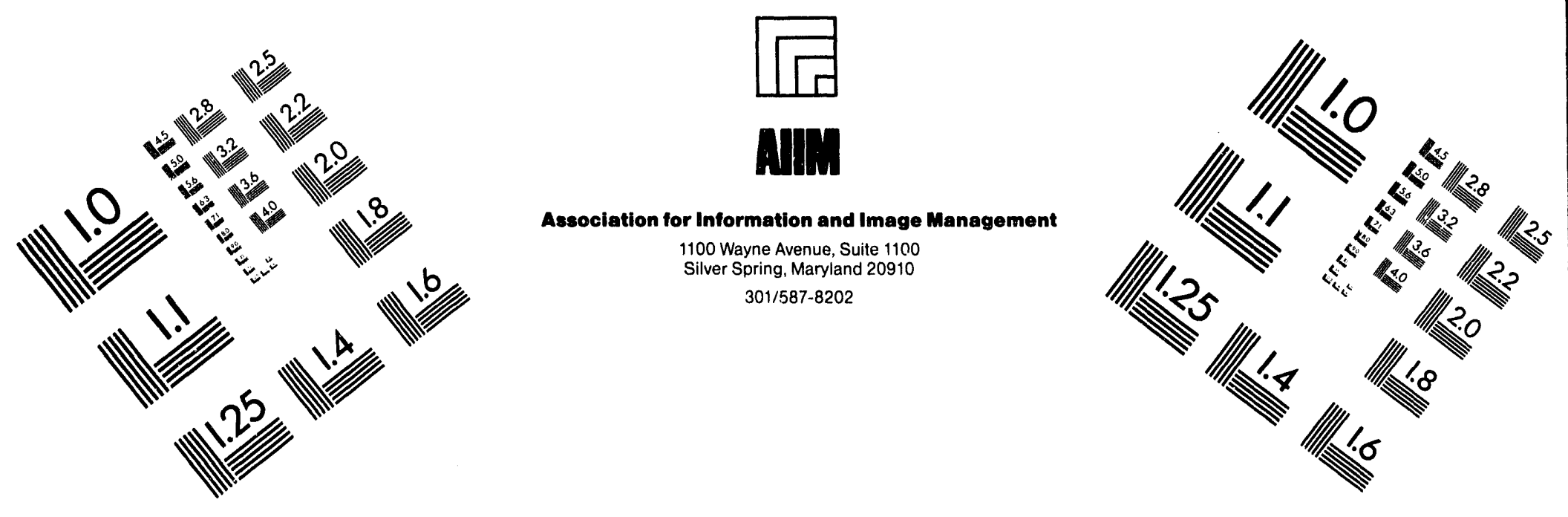

\title{
Centimeter
}

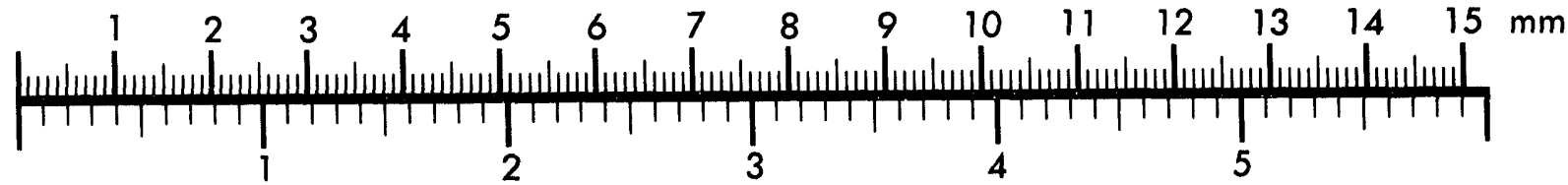
Inches
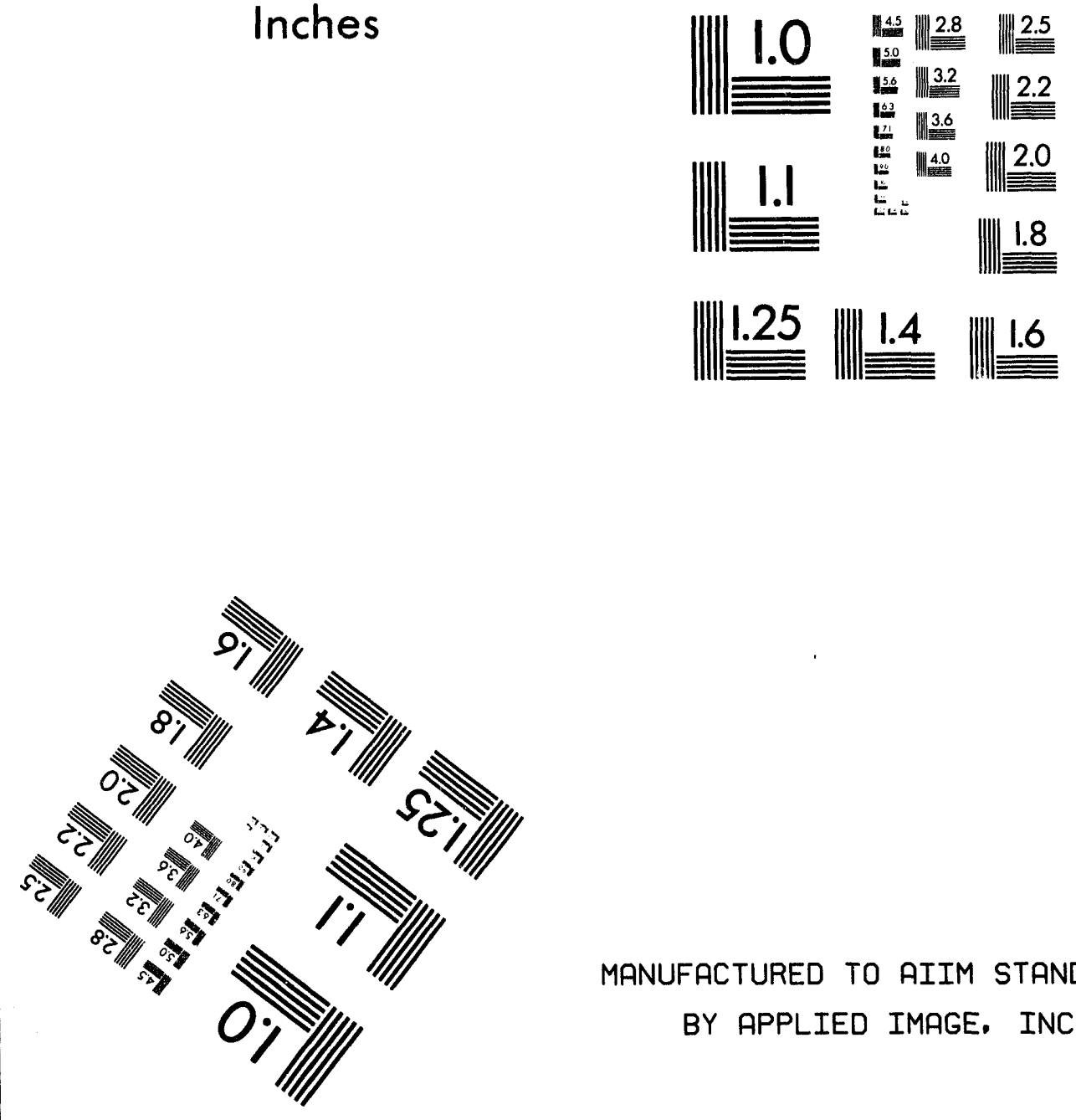

MANUFACTURED TO AIIM STANDARDS

BY APPLIED IMAGE. INC.

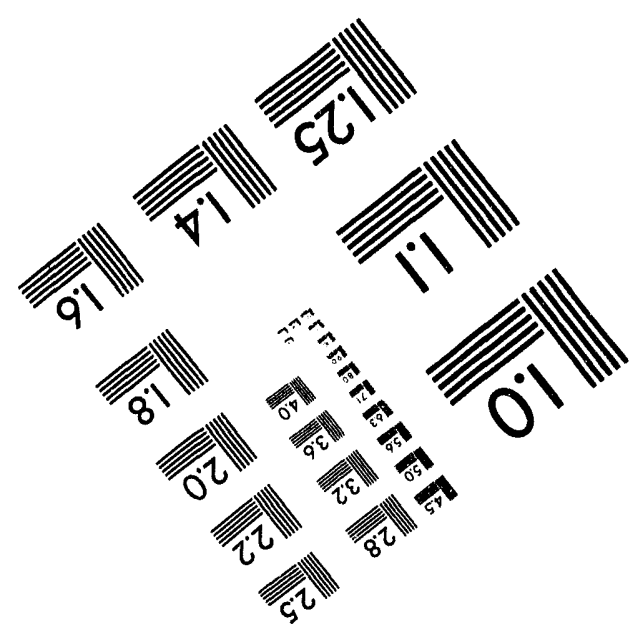



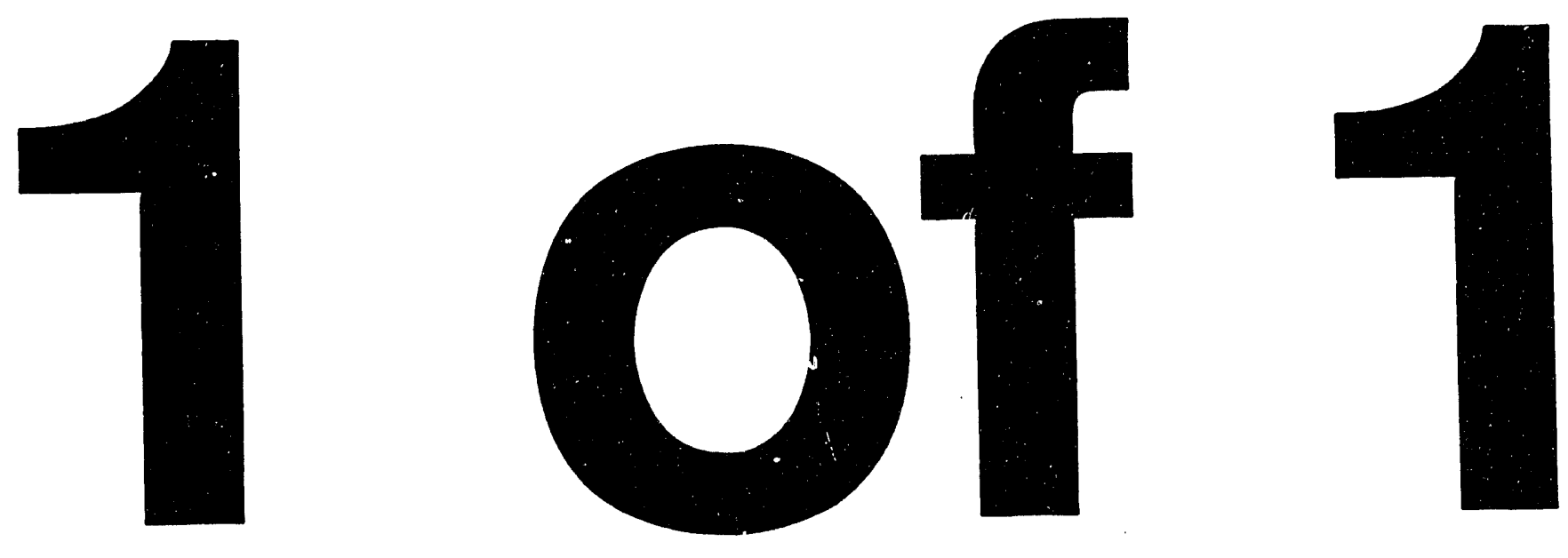


\title{
SANO9+.1804C
}

\section{AN ATOMIC VIEW OF SURFACE DIFFUSION ON METAL SURFACES}

\author{
G. L. Kellogg \\ Sandia National Laboratories \\ Albuquerque, NM 87185 0344
}

\section{Introduction}

The dynamical behavior of individual atoms on single-crystal surfaces is a subject of considerable current interest. A fundamental understanding of how atoms migrate across surfaces and interact with each other is essential to the development of realistic models of thin-film growth, surface alloying, and heterogeneous catalysis. In general, there are two experimental approaches to the study of surface diffusion. Details of the different methods are given in a recent review article by Gomer [1]. The first involves observations of changes in a pre-established concentration gradient of the diffusing species as the system approaches equilibrium [2]. One measures the net transfer of material over a surface in a direction that decreases the free energy of the system. The diffusion coefficient is determined from an analysis based on Fick's second law. A wide range of surface analytical techniques may be employed for the measurements, which makes the method applicable to investigations of self-diffusion or adsorbate diffusion on surfaces of essentially any solid miterial. However, the extraction of quantitative diffusion parameters is difficult because the concentration of diffusing species changes as a function of time, and the effects of adatom-adatom interactions are generally unknown. There is also the possibility of surface defects that can have a significant effect on the transport rate. These problems can be avoided to a certain extent by keeping the net concentration fixed and measuring the time correlation of concentration fluctuations [3], but adatom-adatom interactions may still be present.

The second approach involves direct measurements of the movements of an isolated atom on a single-crystal substrate [4]. Data analysis is simple in the extreme as the diffusion constant is determined directly from the rate of the atom's displacements. Adatom-adatom interactions are completely eliminated (unless one wishes to study them), and the substrate may be prepared to atomic perfection. The main limitation of this approach is that it requires an experimental probe that can resolve individual atoms on a surface. One such instrument is the scanning tunneling microscope (STM) [5], and recent investigations show considerable promise for obtaining quantitative information on single-atom surface diffusion [6]. However, the primary experimental tool used in single-atom surface diffusion studies is an instrument that pre-dates the STM by about 30 years - the field ion microscope (FIM) [7]. In fact, much of our current understanding of how individual atoms move and interact on single-crystal surfaces come from studies with the FIM [8].

It is interesting that the first FIM surface diffusion study was reported nearly 30 years ago in a landmark paper by Ehrlich and Hudda [4]. In the intervening years researchers have applied the experimental methods developed in these early studies to a 
variety of adatom-substrate combinations - mostly metals on metals. The investigations have expanded from studies of single-atom surface diffusion to more complicated processes involving various atom-atom and atom-defect interactions (cluster nucleation is an example). Often, these studies have reinforced our intuitive notions about atomic-level processes at surfaces, i.e., that atom motion takes place by a series of hops between adjacent binding sites on a surface and that cluster nucleation can be explained by simple pair interactions between neighboring atoms. More recently, however, FIM studies have yielded results counter to these intuitive pictures. The discovery of surface diff sion by exchange-mediated mechanisms [9-12] and the observation of atomic-chain configurations during the growth of small clusters [13-16], for example, have forced us to take a closer look at the physics underlying surface diffusion and cluster nucleation. In some cases it makes more sense to view surface diffusion and cluster nucleation in terms of concepts derived from chemistry concerted reactions, bond making and bond breaking, etc. [17]. It is upon these more recent, unexpected observations that $I$ will concentrate in this review of surface diffusion on metal surfaces.

\section{Experimental Procedures}

\subsection{OBSERVATIONAL METHODS}

The experimental procedure used in FIM surface diffusion studies is illustrated by the series of field ion micrographs shown in Fig. 1. The photographs show direct, realspace images of a $\mathrm{Rh}$ surface in the region of a (311) plane. The nearly circular ring of spots is an image of the atoms at the plane's edge. Due to the nature of the image formation process in the FIM, only the edge atom of the low-index planes are imaged, the interior of the plane appears uniformly dark. Just beyond the plane edge there is a step down in all directions. The high-contrast spot within the circular region shown in Fig. 1(a) is an Ir adatom that has been vapor deposited on to the top of the plane. The (311) surface of an fcc metal is a corrugated surface consisting of close-packed rows of atoms with intervening surface channels. Analysis of the position of the adatom with respect to the edge atoms indicate that the Ir adatom lies within one of the surface channels.

The FIM images shown in Fig. 1 are recorded with the sample at $77 \mathrm{~K}$. At this low temperature the Ir adatom and all of the Rh surface atoms are immobile. To study diffusion of the adatom, the applied electric field used to image the surface is turned off and the sample temperature is increased for a fixed interval of time. At the end of the heating interval, the sample is re-cooled to the base temperature of $77 \mathrm{~K}$ and another image is recorded. Between each photograph shown in Fig. 1(a)-(c), the sample is warmed to a temperature of $180 \mathrm{~K}$ for 30 seconds The $\mathrm{Rh}$ substrate atoms are still immobile at this temperature, but the Ir adatom moves from one side of the plane to the other. Continued observations like those in Fig. 1(a)-(c) indicate that the adatom performs a one-dimensional random walk with the edge of the plane acting as a reflecting boundary. The presence of a reflection boundary, which is present for many (but not all) adatom-substrate combinations, allows one to make tens to hundreds of observations of the adatom as it diffuses back and forth across the surface [8]. 

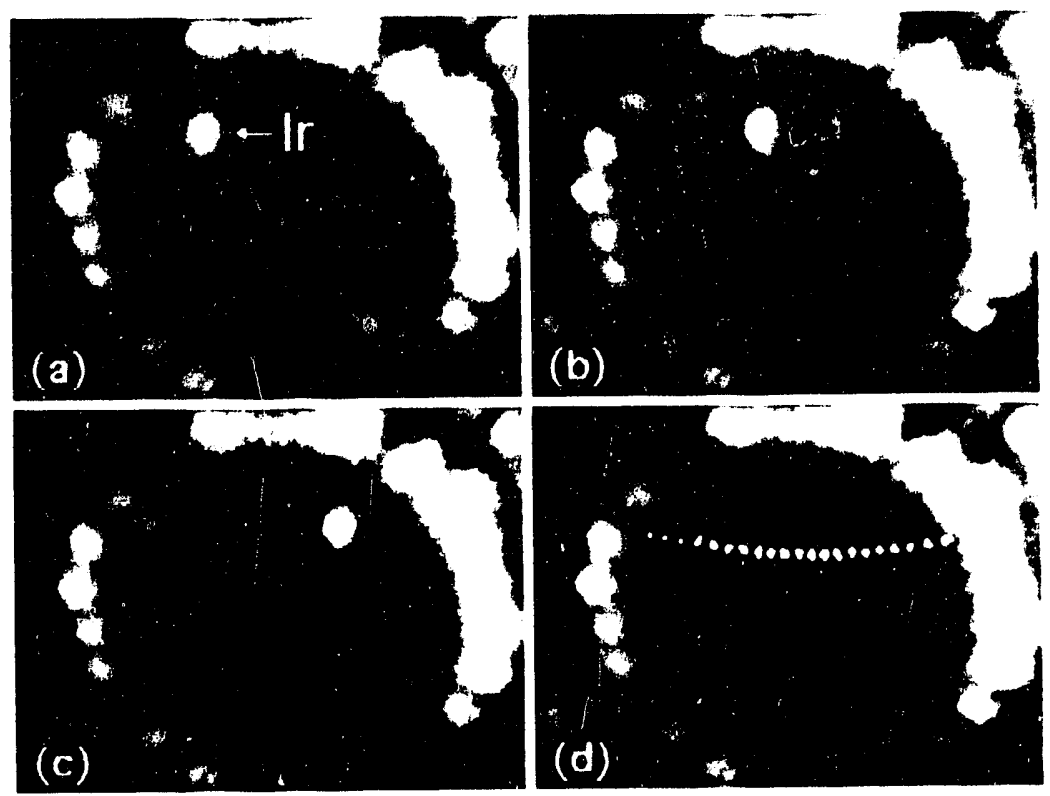

Figure 1 Field ion micrographs illustrating the experimental procedures used in FIM studies of single-atom surface diffusion

The one-dimensional nature of the diffusion is made clear by the photograph shown in Fig. 1(d). This image is from the same $\mathrm{Rh}(311)$ plane, but with the Ir adatom removed. Superimposed on the image are small white dots corresponding to the coordinates of the adatom after each of 300 diffusion intervals. The discrete clusters of dots seen in Fig. 1(d) correspond to the individual binding sites that the atom visits as it moves across the surface. The sites clearly fall on the same line indicating that the diffusing adatom remains within a given surface channel of the (311) plane. The deviation from a perfect straight line is due to the non-uniform magnification of the FIM [18]. Binding-site maps such as the one shown in Fig. 1(d) are very useful in that they allow one to calibrate the distance scale on the FIM image exactly at the position where the atom is moving. They are also used to identify the mechanism of surface diffusion on certain surfaces as discussed in section 3.2 .

\subsection{DATA ANALYSIS}

From observations such as those shown in Fig. 1, one can measure the length of the adatom's displacements during each heating interval. The actual number of jumps that the adatom makes in a given interval is not known - only its net displacement. Thus, in FIM surface diffusion studies data analysis in not based on actual displacement rates, but rather on the mean-square displacement of the adatom during the diffusion interval. These values are typically determined at a series of temperatures, limited by the onset of adatom motion (lower limit) and the frequent loss of adatoms off the plane (upper limit). The equation that relates the measurable parameters (i.e., the mean-square 
displacement and the temperature) to the quantities of interest (i.e., the activation energy of surface diffusion and atomic vibrational frequency) is

$$
<x^{2}>/ 2 \tau=D_{0} \exp \left(-E_{d} / k T\right)
$$

where $\left\langle x^{2}\right\rangle$ is the mean-square displacement in one dimension, $\tau$ is the diffusion time interval, $E_{d}$ is the activation energy for surface diffusion, $k$ is Boltzmann's constant, and $T$ is the temperature. $D_{0}$ is a prefactor term that is roughly equal to the atomic vibrational frequency times the jump length. Derivations of equation (1) along with detailed discussions of the contribution of entropy effects to $D_{0}$, corrections due to the reflecting boundaries, the presence of driving forces, etc. may be found in a number of more extensive review articles [8].

To obtain the diffusion parameters, one simply makes a standard Arrhenius plot of in $\left\langle x^{2}>/ 2 \tau\right.$ vs. 1/T and determines the activation energy and prefactor from the slope and intercept, respectively. On surfaces where diffusion is not restricted to one-dimension, the analysis is essentially the same as above except that $\left\langle x^{2}\right\rangle$ in equation (1) is replaced by $\left\langle r^{2}\right\rangle=\left\langle x^{2}\right\rangle+\left\langle y^{2}\right\rangle$. In either case the accuracy of the activation energy measurement is about $\pm 5 \%$. The Arrhenius prefactor is reliable only to within an order of magnitude. From measurements taken over a large variety of adatom-substrate combinations, it is now generally agreed that the prefactor is essentially invariant from system to system and is given by $-10^{-3} \mathrm{~cm}^{2} / \mathrm{sec}$ [19]. It is therefore possible to calculate the activation energy for surface diffusion from measurements of the mean-square displacement at a single value of the temperature. For some adatom-substrate combinations this is necessary because the temperature range over which the experiments can be performed is too small to carry out a full Arrhenius analysis.

\section{Selected Experimental Results}

\subsection{CONVENTIONAL HOPPING DIFFUSION}

Most of the early FIM investigations of single-atom surface diffusion were concerned with metal adatoms migrating on the (110) plane of $\mathrm{W}$, primarily because the cleaning and imaging procedures for $\mathrm{W}$ were well established. Over the years the data base for diffusion on $W(110)$ has been extended to other adatoms - even silicon [20]. Although a detailed discussion of these results is beyond the scope of this article, the measured activation energies of surface diffusion for various adatoms on the W(110) plane provide a nice summary of the general trends associated with single-atom diffusion on metal surfaces. In Fig. 2 the activation energies of surface diffusion for nine elements are plotted against the bulk sublimation energies of the adatoms. A better comparison would be to plot the activation energies of surface diffusion against the binding energies of the adatoms on W(110), but these values have not all been measured. Where they have, the binding energies are found to be of the same order of magnitude as the sublimation energies (several eV) and follow the same trend of increasing energy with increasing melting point [21]. The plot in Fig. 2 thus demonstrates two of the "rules of thumb" associated with diffusion on metal surfaces: (1) surface diffusion activation energies are about $10 \%$ of adatom binding energies and 
(2) on a given substrate, the activation energy for surface diffusion is higher for the higher melting point elements.

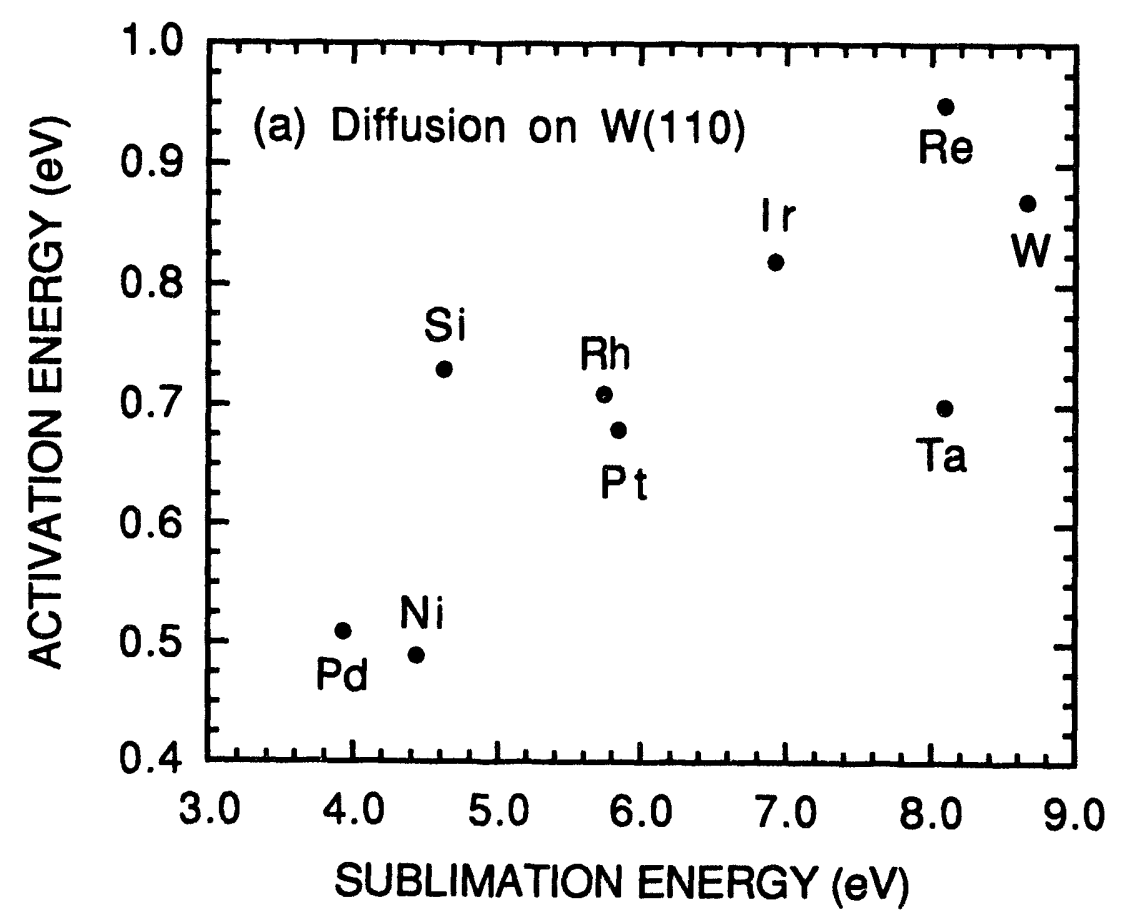

Figure 2 Activation energies of surface diffusion for single atoms on the W(110) plane plotted as a function of the bulk sublimation energy of the diffusing species. References to the original measurements can be found in recent review articles [8]

The scaling of the activation energies of surface diffusion with binding energies fits well with the intuitive notion that an adatom migrates across a surface by a series of hops between adjacent binding sites. An adatom more tightly bound to the surface resides in a deeper potential well and must overcome a higher potential barrier to diffuse. This simple picture of adatom diffusion is further reinforced by measurements of diffusion barriers as a function of surface atomic structure. For both $\mathrm{Rh}$ and $\mathrm{Pt}$ adatoms migrating on various planes of $\mathrm{Rh}$, the activation energy is quite low on the smooth (111) plane and much higher on the more open (100) plane [22,23]. On the channeled surfaces such as (110), (311), and (331) planes, the motion is confined to one dimension, i.e., along the direction of minimum corrugation. The physical picture of a marble (adatom) rolling over a solid array of marbles (surface atoms) thus appears to adequately describe the results of FIM studies of adatom diffusion on surfaces of W and $\mathrm{Rh}$.

\subsection{EXCHANGE-MEDIATED DIFFUSION}

Probably the biggest surprise to result from FIM studies of surface diffusion was the discovery that adatoms may migrate across a surface by a mechanism other than 
conventional hopping. FIM experiments show conclusively that for certain adatomsubstrate combinations atom motion may proceed by an "exchange" or "replacement" process. Instead of hopping across the surface, the adatom finds it energetically favorable to push a neighboring atom out of the substrate plane and take its place in the top layer of surface atoms. In the case of self-diffusion (adatom and substrate atoms are the same chemical species) the displaced atom becomes a new adatom and continues the migration process. If the adatom and substrate atoms are different chemical species, then the initial exchange displacement produces an adatom of the substrate element, and diffusion of the original adatom cannot be studied. In the latter case the exchange process leads to surface alloying rather than surface diffusion. As shown below, FIM experiments have confirmed the existence of exchange displacements for both surface self-diffusion and surface alloying.

The first atomic-level, experimental evidence for exchange displacements was reported in 1978 by Bassett and Webber [9]. In FIM studies of self-diffusion on $\mathrm{Pt}(110)$, they observed displacements of the Pt adatom both along the surface channels and across the channel walls. In fact, the activation barriers for motion in the two directions were found to be the same within the experimental errors. The observation of cross-channel displacements is totally inconsistent with the conventional picture of metal-on-metal surface diffusion. To explain this unexpected result, they suggested that cross-channel displacements on $\mathrm{Pt}(110)$ are due to an exchange process between the adatom and a channel-wall atom. That such an exchange processes actually take place on fcc(110) surfaces was subsequently confirmed in an investigation of $\mathrm{W}$ adatoms on the $\operatorname{Ir}(110)$ surface using an atom-probe (i.e., a time-of-flight mass spectrometer attached to an FIM). Identification of the adatom appearing after a cross-channel movement provided convincing evidence that an exchange process was involved in the displacement process [10]. More recently, a similar confirmation of exchange processes for $\mathrm{Pt}$ atoms on the $\mathrm{Ni}(110)$ surface has been reported [24]. These studies have led to the reasonable assumption that all cross-channel displacements on $\mathrm{fcc}(110)$ surfaces (including self-diffusion) are due to exchange processes.

For about ten years following the initial work of Bassett and Webber, it was assumed that exchange displacements occurred only on the channeled (110) surfaces of a few fcc metals. The relatively low coordination of atoms in the channel walls was believed to make the exchange process energetically accessible. As a result, the discovery was considered to be fundamentally interesting, but not of much general importance. A considerable amount of renewed interest in exchange diffusion was generated in 1990 when it was shown that a similar exchange process is also energetically favorable on the non-corrugated (100) surfaces of fcc metals. The detailed mechanism involved in exchange displacements on the $\mathrm{fcc}(100)$ plane was first proposed by Feibelman [17] based on first-principles calculations for self-diffusion on $\mathrm{Al}(100)$. Experimental confirmation of the exchange mechanism came shortly thereafter from FIM studies of self-diffusion on the (100) surfaces of $\mathrm{Pt}$ [11] and Ir [12].

The exchange mechanism for adatoms on fcc(100) surfaces is shown schematically in Fig. 3. An adatom in a four-fold hollow begins the displacement by moving down towards a surface atorn. At the same time, the surface atom begins to move up out of its lattice position. At the saddle point of the transition, the adatom and the displaced surface atom have identical bonding configurations above the surface with a vacancy below. The displacement ends when the adatom fills the vacancy and the surface adatom becomes a new adatom at a next-nearest-neighbor position with respect to the original adatom. The calculations predict that the activation barrier for the exchange 
process is about a factor of three smaller than that for hopping over the two-fold bridge site [17]. The reason for the lower barrier is that the adatom can maintain three "bonds" with neighboring atoms in the concerted exchange process. This bonding configuration is particularly attractive for $\mathrm{Al}$ which is trivalent. To hop over a bridge site, the adatom must break one of its bonds with the surface atoms.

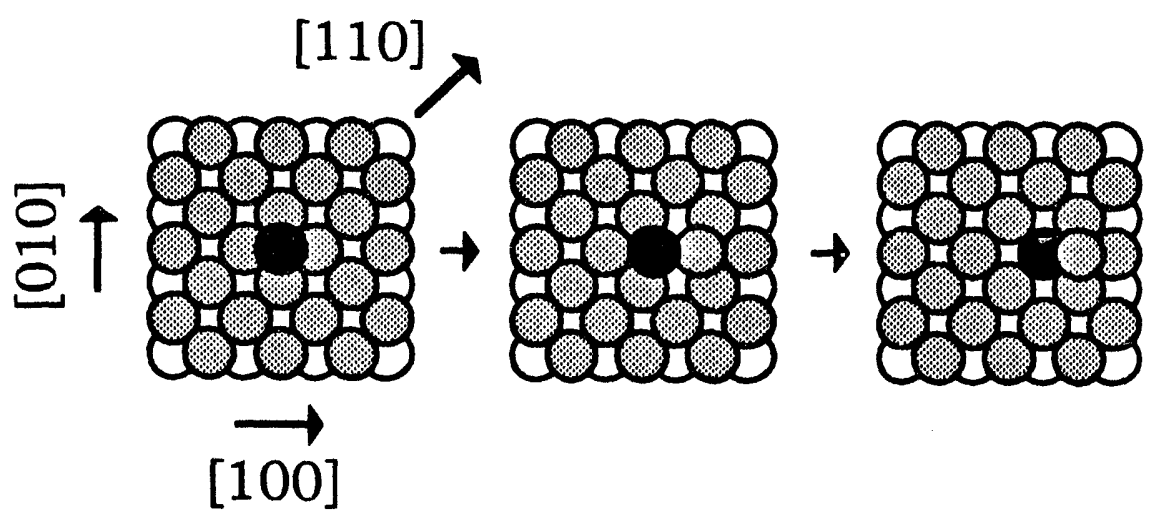

Figure 3 A schematic illustration of the exchange mechanism on fcc(100) surfaces. The shaded circles represent surface atoms of top the (100) layer. The filled circle represents an adatom which becomes incorporated into the surface layer as a result of the exchange process.

Experimental verification of the proposed exchange mechanism relies on the fact that the directions of individual displacements are different for the exchange vs. hopping processes. As shown in Fig. 3, exchange displacements involve the concerted motion of an adatom and a surface atom along the [100] and [010] directions, i.e., displacements to next-nearest neighbor positions. Hopping displacements involve motion along the [110] and equivalent directions, i.e., displacements to nearest neighbor positions. If diffusion proceeds exclusively by exchange displacements, then the adatom can visit only half of the available surface sites. The map of sites visited by the adatom is a square pattern with sides parallel to the [100] and [010] directions. In the notation of surface overlayers, the map has $c(2 \times 2)$ periodicity. The situation is analogous to playing the game of checkers in which the game piece is permitted to move only in diagonal directions and is therefore confined to squares of the same color. If diffusion proceeds by ordinary hopping, then all of the surface sites may be visited. The map of visited sites for hopping displacements is a $(1 \times 1)$ square pattern with sides parallel to the [110]-type directions.

Field ion microscope images showing the diffusion of a $\mathrm{Pt}$ adatom on a $\mathrm{Pt}(100)$ plane along with the resulting site-visitation map are presented in Fig. 4 . The first three photographs show the position of the adatom after 30 -sec. diffusion intervals at a temperature of $175 \mathrm{~K}$. A small white dot is superimposed on the images to indicate the adatom's position in the current and previous images. Motion of the adatom is evident from the location of the dots in Fig. 4(b)-(c). With continued heating cycles the sitevisitation map fills in and eventually produces the pattern shown in Fig. 4(d). This photograph was taken after 300 diffusion intervals. The orientation of the 
crystallographic directions is obtained from the symmetry of the overall field ion image. The map of sites is clearly a square pattern with sides parallel to the [100]-type directions. This result [11] and similar observations on $\operatorname{Ir}(100)$ [12] provides compelling (albeit indirect) evidence that self-diffusion on the (100) surfaces of $\mathrm{Pt}$ and Ir takes place by exchange displacements.
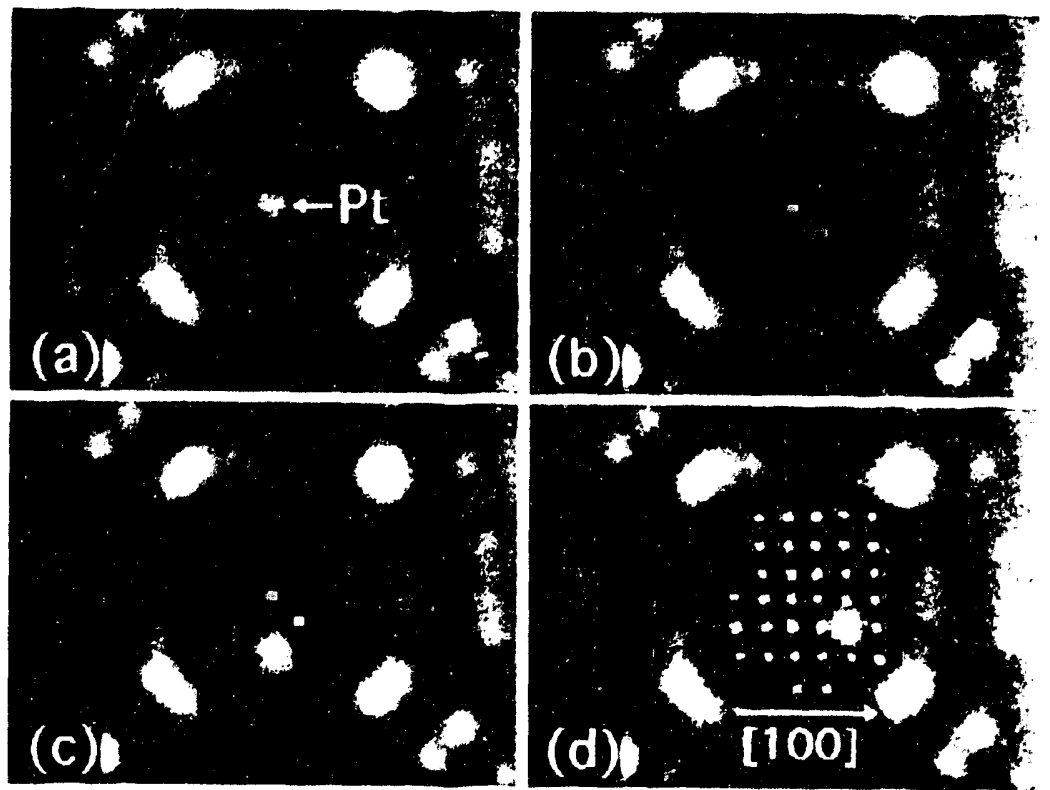

Figure 4 A series of field ion micrographs illustrating the confirmation of the exchange mechanism for self-diffusion on $\mathrm{Pt}(100)$ as discussed in the text.

A more direct confirmation of the exchange mechanism on $\mathrm{fcc}(100)$ surfaces is obtained from FIM studies of Ir adatoms on the (100) plane of Rh [25]. Examples of FIM images from this experiment are shown in Fig. 5. The bright spot near the center of the (100) plane shown in Fig. 5(a) is an Ir atom, identified by its high desorption field (i.e., the electric field required to remove the atom from the surface). Whereas Ir adatoms resist field desorption at electric fields up to the substrate evaporation field (see below), $\mathrm{Rh}$ adatoms desorb at about $75 \%$ of this value. The same $\mathrm{Rh}(100)$ surface is shown in Fig. 5(b) following a 30-sec. heating interval at $330 \mathrm{~K}$. An adatom is present at about the same position as the one in Fig. 5(a), but a careful analysis of the two images indicates that the adatom has made a small displacement. More importantly, the adatom in Fig. 4(b) desorbs from the surface at an electric field strength corresponding to $\mathrm{Rh}$, not Ir. This indicates that an exchange process has occurred producing a $\mathrm{Rh}$ adatom and embedding the Ir adatom $R h$ surface layer.

The $\mathrm{Rh}(100)$ plane with the $\mathrm{Rh}$ adatom removed is shown in Fig. 4(c). The Ir atom, now embedded in the top layer of surface atoms, is not visible because it does not protrude out from the surface. However, the Ir-Rh exchange process may be further confirmed by field evaporation of the top layer of $\mathrm{Rh}(100)$ surface atoms. Field evaporation is the removal of the substrate surface atoms by a high electric field. In the 
region of the low-index crystal planes, this process removes atoms from the edge of the plane inward in a controlled, layer-by-layer fashion. Figs. 4(d)-(f) show the $R h(100)$ surface at successive stages of field evaporation. It is clear in Fig. 4(f) that when the $\mathrm{Rh}(100)$ top-layer atoms are removed, an individual adatom remains on the surface. From its resistance to field desorption, this adatom is identified as Ir - the same Ir adatom that embedded itself into the surface in the exchange process. The sequence shown in Fig. 4 can be repeated over and over. Each time the surface is heated, a Rh adatom is generated and each time the $\mathrm{Rh}(100)$ surface is field evaporated, the Ir adatom reappears.

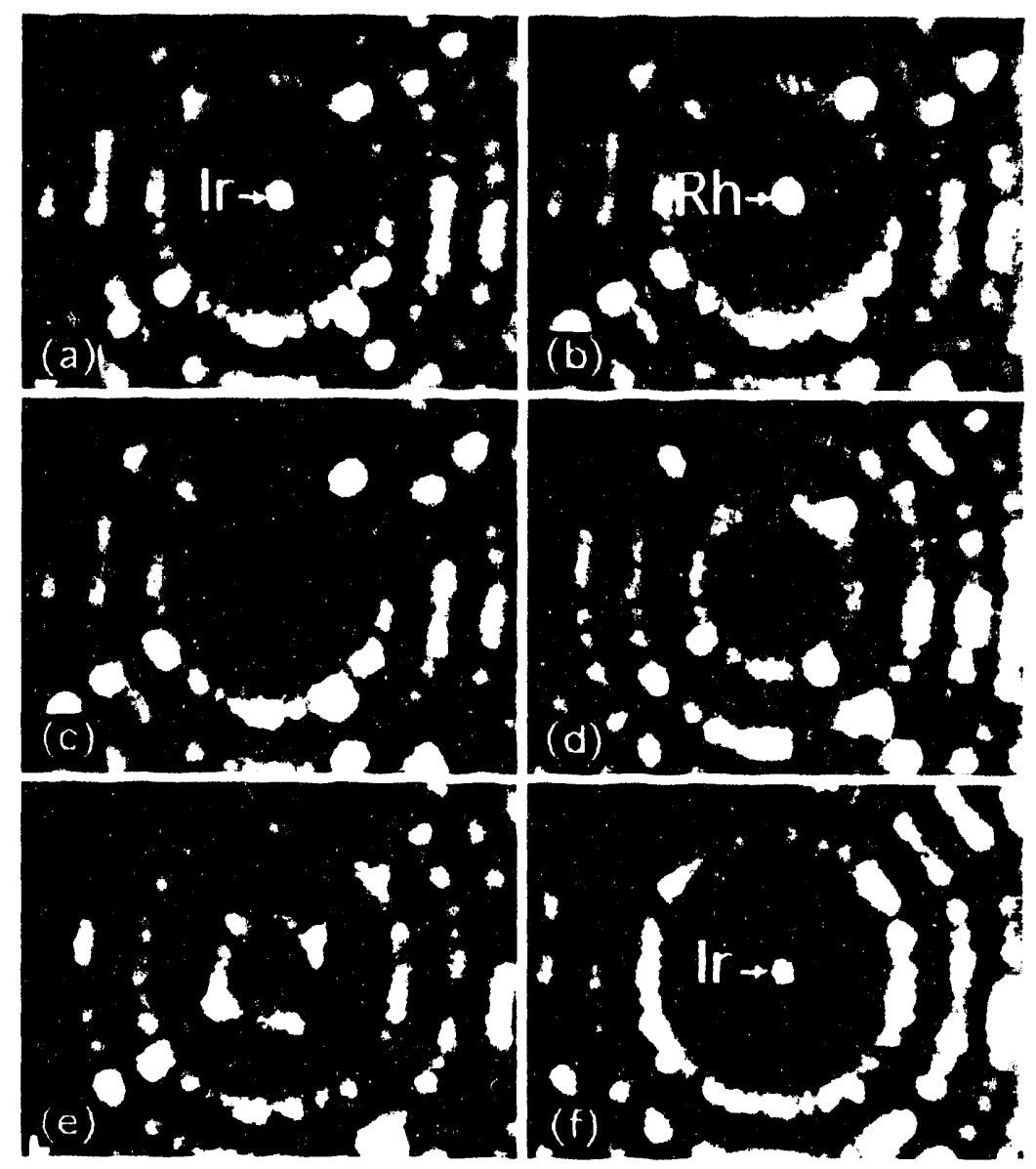

Figure 5 Field ion micrographs illustrating the exchange mechanism for an Ir adatom on a $\mathrm{Rh}(100)$ plane. From ref. [25].

The general agreement between the theoretical predictions of a lower activation barrier for exchange processes [17] and the above experiments provide compelling evidence that exchange displacements are energetically favorable over hopping displacements on certain fcc(100) surfaces. In addition to is fundamental significance as a new means of atom transport and surface alloying, the discovery of the exchange 
mechanism has interesting practical implications. For example, it has been suggested that one may be able to produce patterned, buried interfaces with atomic-layer dimensions using the exchange process [26]. On the negative side, exchange displacements may have a deleterious effect in the fabrication of layered materials such as $x$-ray optics where atomically sharp interfares are desired. In recent investigations of epitaxial growth on the (100) surfaces of $\mathrm{P}, \mathrm{u}, \mathrm{Ag}$, and $\mathrm{Cu}$ (some related to thin-film magnetism) exchange displacements are 'uspected to be important in defining the nature of the growth process and controlling the resulting morphology of the film [2731].

In view of these fundamental and practical implications, it is important to establish how commonly exchange displacemen's occur. Additional FIM studies indicate that exchange displacements are preferred for some adatom-substrate combinations and hopping displacements for others. Exchange displacements also occur in the migration of small clusters of $\mathrm{Pt}$ on $\mathrm{Pt}(100)$ [32]. However, the systems for which exchange displacements occur do not seem to be correlated with macroscopic bulk or surface properties of the materials [23]. There is some evidence that the degree of surface relaxations induced by the adatom is correlated with the propensity for exchange [33]. This possibility is consistent with the effect of an external clectric field on the rate of exchange displacements [34], but more systems need to be examined. The current challenge is to define the laws that govern whether adatoms on a given substrate will move by exchange or hopping displacements. Further experimentation and more extensive theoretical modeling are currently underway to address this challenge.

\subsection{ADATOM-ADATOM INTERACTIONS AND CLUSTER NUCLEATION}

The single-atom surface diffusion studies discussed above represent only a small fraction of the contributions made by the FIM to our understanding of atomic processes on surfaces. Similar methods have been used to investigate the diffusion behavior of two or more atoms on a crystal plane. Detailed measurements of the pair distribution functions of interacting adatoms and dissociation lifetimes of small clusters have produced quantitative values for the interatomic potentials between adatoms on surfaces. The interaction of diffusing atoms with steps and other surface defects has also been studied extensively with the FIM. Often, the interactions energies are found to be non-monotonic or oscillatory in nature, suggesting the presence long-range electronic or elastic interactions on surfaces. The interested reader is referred to several review articles for detailed discussion of these investigations [8].

In this brief review I discuss a somewhat different approach used in FIM investigations of adatom-adatom interactions on surfaces. As adatoms nucleate into small clusters, FIM observations show that the stable configuration of atoms is not always the expected two-dimensional island. For some systems the clusters are found to be more stable as one-dimensional chains of atoms [13,14,35]. A particularly interesting example is the nucleation of Ir clusters on the $\operatorname{Ir}(100)$ plane [15]. FIM studies show conclusively that clusters consisting of five atoms or less are more stable as one-dimensional chains than two dimensional islands. For six atoms or more, the situation is reversed. Pt clusters on the $\mathrm{Rh}(100)$ surface exhibit a similar behavior. A simple lattice gas model indicates that in order to explain these configurational stabilities, interactions extending beyond second-nearest neighbors, many body interactions, or both must be invoked [36]. This is an important observation as most 
simulations of growth still assume that adatom-adatom interactions can be modeled by pair interactions between neighboring atoms.

Even more interesting is the system of $\mathrm{Pt}$ on $\mathrm{Pt}(100)$. Here, the stable configuration oscillates between a chain and an island as the cluster increases in size from three to six atoms [16]. This behavior is observed in FIM experiments and total energy calculations using embedded atom method (EAM) potentials. Interestingly, the calculations give the correct result only when the substrate surface atoms are allowed to relax in the presence of the cluster atoms. Thus, the response of the substrate atoms to the presence of the adatoms plays an important role in cluster nucleation processes - the surface atoms are not simply a static template on which the adatoms move.

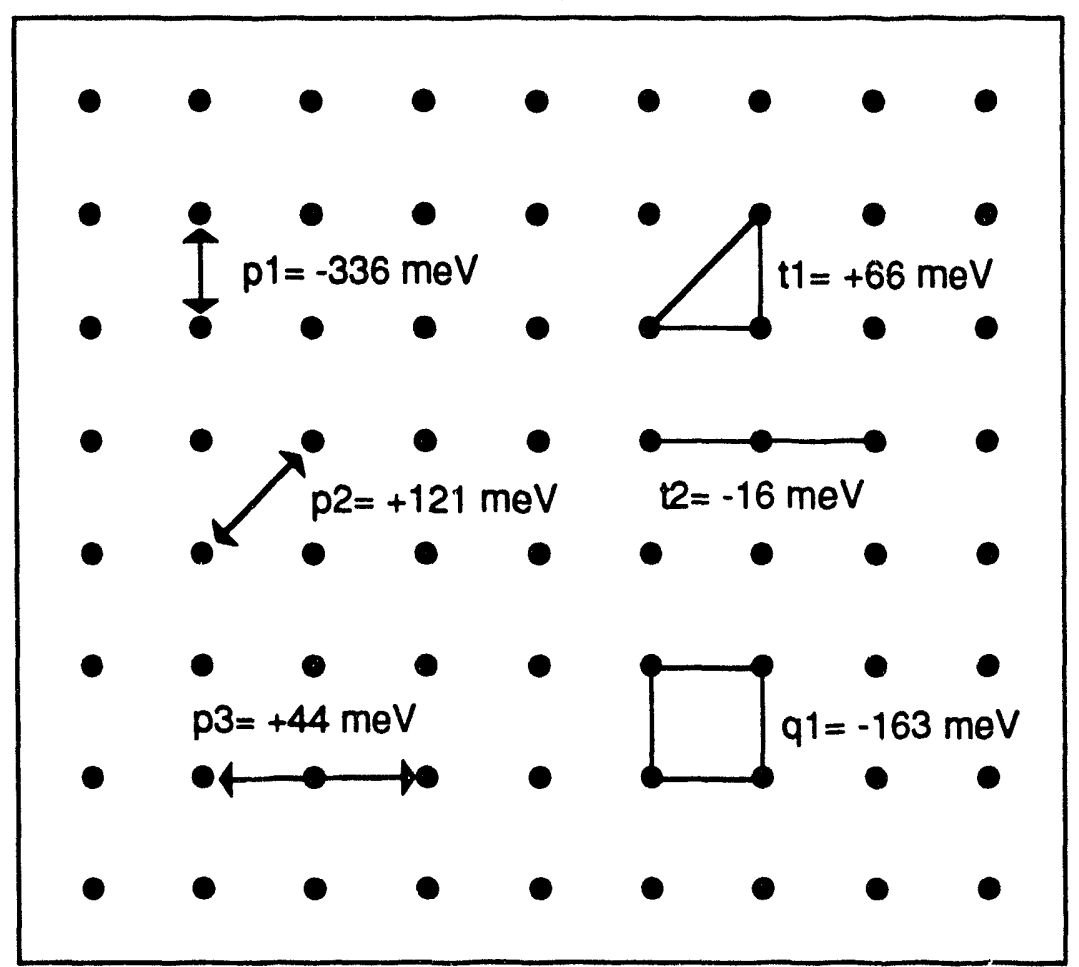

Figure 6 Adatom-adatom interactions energies for $\mathrm{Pt}$ on $\mathrm{Pt}(100)$ as determined from embedded atom method calculations [16]. pl, 2, etc. are pair energies between atoms at the distances indicated; 11 and 12 are triplet interactions as indicated; and q1 is the quadruplet interaction four for atoms in a square.

The agreement between the total energy calculations and the experimental observations in predicting the configurational stabilities also gives one confidence that the adatom-adatom interaction energies, which can be extracted from the calculations, are valid. The actual values are shown in Fig. 6 . Note that pair interactions between third-nearest neighbors and many-body interactions (up to four-body) contribute to the total energy of the cluster. The oscillatory nature of the configurational stability can be rationalized by these interaction energies. Chain structures for three and five atoms 
result from a rather large repulsive interaction between next-nearest neighbors and a large repulsive three-body interaction for atoms in the triangular configuration. The island structure is stabilized for clusters of four, six, and more atoms by the surprisingly large four-body interaction for atoms in a square.

It should be noted that one may obtain adatom-adatom interaction energies from direct measurements of the diffusion behavior of two or more atoms on a surface, as mentioned above. However, the number of observations required to obtain statistically significant results for even two atoms on a surface already taxes current data gathering methods. To probe three-body or higher-order interactions is beyond present day capabilities. Examination of configuratioital stabilities combined with theoretical calculations thus provites an alternative approach to investigate the details of adatomadatom interactions during cluster nucleation and allows one to identify the important physical phenomena (lattice relaxations, many-body interactions, et.) involved in the nucleation process.

\section{Summary}

Investigations of surface diffusion and cluster nucleation by field ion microscopy have provided a considerable amount of physical insight concerning the fundamental interactions that control dynamical processes on surfaces. The investigations rely not only on the FIM's ability to resolve and track individual atoms on a surface, but also its ability to manipulate the number of adatoms and the size of clusters by the process of field desorption. The results of the investigations are often quite surprising. Whereas metal atom diffusion was once thought to be a simple, and perhaps uninteresting hopping process, FIM experiments have revealed new mechanisms for atom transport. Whereas cluster nucleation was once thought to be an aggregation process dependent only upon pairwise interactions between atoms, FIM investigations have shown that long-range and many body interactions can make non-negligible contributions to the overall process. By providing a brief overview of the experimental methods used in FIM surface diffusion studies and discussing a few selected applications, I hope to have conveyed some of the rich history as well as the current excitement associated with FIM investigations of dynamical processes on surfaces.

\section{Acknowledgment}

Work performed at Sandia National Laboratories was supported by the U. S. Department of Energy under contract no. DE-AC04-94AL85000.

\section{References}

1. Gomer, R. (1990) Diffusion of Adsorbates on Metal Surfaces, Rep. Prog. Phys. 53, 917-1002.

2. Blakely, J. M. (1963) Surface Diffusion, Progress in Materials Science 10, 395-437.

3. Gomer, R. (1973) Current Fluctuations from Small Regions of Adsorbate Cuvered Field Emitters, Surf. Sci. 38, 373-393. 
4. Ehrlich, G. and Hudda, F. G. (1966) Atomic View of Surface Diffusion: Tungsten on Tungsten, J. Chem. Phys. 44, 1039-1049.

5. Binnig, G., Rohrer, H., Gerber, C., and Weibel, E. (1982) Surface Studies by Scanning Tunneling Microscopy, Phys. Rev. Lett. 49, 57-61.

6. See: Lagally, M. G. (1993) Atom Motion on Surfaces, Physics Today 46, 24-31 and references therein.

7. Müller, E. W. (1951) Das Feldionenmikroskop, Z. Phys. 131, 136-142.

8. For recent reviews, see: Bassett, D. W. (1983) Observing Surface Diffusion at the Atomic Level: I. Single Atoms. in V. T. Binh (ed.), Surface Mobilities on Solid Materials , Plenum, New York, pp. 63-82; Tsong, T.T. (1990) Atom-Probe Field Ion Microscopy, Cambridge University Press, Cambridge, pp. 202-265; Ehrlich, G. (1990) Direct Observation of Surface Diffusion, Scanning Microscopy 4, 829-842; Kellogg G.L. (1994) Field Ion Microscope Studies of Single-Atom Diffusion and Cluster Nucleation on Metal Surfaces, Surf. Sci. Rep. (in press).

9. Bassett, D. W. and Webber, P. R. (1978) Diffusion of Single Adatoms of Platinum, Iridium, and Gold on Platinum Surfaces, Surf. Sci. 70, 520-531.

10. Wrigley, J. D. and Ehrlich, G. (1980) Surface Diffusion by an Atomic Exchange Mechanism, Phys. Rev. Lett. 44, 661-663.

11. Kellogg, G. L. and Feibelman, P. J. (1990) Surface Self-Diffusion on Pt(100) by an Atomic Exchange Mechanism, Phys. Rev. Lett. 64, 3143-3146.

12. Chen, C. L. and Tsong, T. T. (1990) Displacement Distribution and Atomic Jump Direction in Diffusion of Ir Atoms on the Ir(001) Surface, Phys. Rev. Lett. 64, 31473150.

13. Bassett, D. W. (1978) Field Ion Microscope Studies of Submonolayer Films of Nickel, Palladium, and Platinum on (110) Tungsten Surfaces, Thin Solid Films 48, 237 246.

14. Fink, H.-W. and Ehrlich, G. (1981) Direct Observation of Overlayer Structures on W(110), Surf. Sci. 110, L611-L614.

15. Schwoebel, P. R. and Kellogg, G. L. (1988) Structure of Iridium Adatom Cluster Nuclei on $\operatorname{Ir}(100)$, Phys. Rev. Lett. 61, 578-580.

16. Schwoebel, P. R., Foiles, S. M., Bisson, C. L., and Kellogg, G. L. (1989) Structure of Platinum Adatom Clusters on $\mathrm{Pt}(100)$ : Experimental Observations and Embedded Atom Method Calculations, Phys. Rev. B 40, 10639-10642.

17. Feibelman, P. J. (1990) Diffusion Path for an Al Adatom on Al(001), Phys. Rev. Lett. 65, 729-732.

18. Müller, E. W. and Tsong, T. T. (1969) Field Ion Microscopy, Principles and Applications American Elsevier, New York.

19. Wang, S. C. and Ehrlich, G. (1988) Adatom Diffusion on W(211): Re, W, Mo, Ir, and $\mathrm{Rh}$, Surf. Sci. 206, 451-474.

20. Casanova, R. and Tsong, T. T. (1981) Direct Observations of the Behavior of Single Silicon Atoms on a Metal Surface, Surf. Sci. 109, L497-L503.

21. Berlowitz, P. J. and Goodman, D. W. (1987) The Adsorption of $\mathrm{H}_{2}$ and $\mathrm{CO}$ on Strained Ni Overlayers on W(110) and W(100), Surf. Sci. 187, 463-480; Berlowitz,P.J. and Goodman,D.W. (1988) Chemisorption of Ultrathin Pd Layers on W(110) and W(100): Adsorption of $\mathrm{H}_{2}$ and $\mathrm{CO}$, Langmuir 4, 1091-1095; Schlenk, W. and Bauer, E. (1980) Properties of Ultrathin Layers of Palladium on A Tungsten (110) Surface, Surf. Sci. 93, 9-32.

\section{DISCLAIMER}

This report was prepared as an account of work sponsored by an agency of the United States Government. Neither the United States Government nor any agency thereof, nor any of their employees, makes any warranty, express or implied, or assumes any legal liability or responsibility for the accuracy, completeness, or usefulness of any information, apparatus, product, or process disclosed, or represents that its use would not infringe privately owned rights. Reference herein to any specific commercial product, process, or service by trade name, trademark, manufacturer, or otherwise does not necessarily constitute or imply its endorsement, recommendation, or favoring by the United States Government or any agency thereof. The views and opinions of authors expressed herein do not necessarily state or reflect those of the United States Government or any agency thereof. 
22. Kellogg, G. L. (1991) Direct Observation of Adatom-Surface Atom Replacement: Pt on Ni(110), Phys. Rev. Lett. 67, 216-219.

23. Kellogg, G. L. (1994) Direct Observation of Substitutional-Atom Trapping on a Metal Surface, Phys. Rev. Lett. 72, 1662-1665.

24. Tsong, T. T. (1991) Effects of an Electric Field in Atomic Manipulations, Phys. Rev. B 44, 13703-13710.

25. Schmitz, P. J., Leung, W.-Y., Graham, G. W., and Thiel, P. A. (1989) Novel MetalFilm Configuration: $\mathrm{Rh}$ on $\mathrm{Ag}(100)$, Phys. Rev. B 40, 11477-11487.

26. Egelhoff Jr., W. F. (1991) X-Ray Photoelectron and Auger Electron ForwardScattering Studies of the Epitaxial Growth of $\mathrm{Fe}$ on $\mathrm{Ag}(100)$, Proc. Mat. Res. Soc. 229, 27.

27. Rousset, S., Chiang, S., Fowler, D. E., and Chambliss, D. D. (1992) Intermixing and Three-Dimensional Islands in the Epitaxial Growth of $\mathrm{Au}$ on $\mathrm{Ag}(110)$, Phys. Rev. Lett. 69, 3200-3203.

28. He, Y.-L. and Wang, G.-C. (1993) Observation of Atomic Place Exchange in Submonolayer Heteroepitaxial Fe/Au(001) Films, Phys. Rev. Lett. 71, 3834-3837.

29. Johnson, K. E., Chambliss, D. D., Wilscn, R. J., and Chiang, S. (1993) Growth and Morphology of Partial and Multilayer Fe Thin Films on $\mathrm{Cu}(100)$ and the Effect of Adsorbed Gases Studied by Scanning Tunneling Microscopy, J. Vac. Sci. Technol. A 11, 1654-1660.

30. Kellogg, G. L. and Voter, A. F. (1991) Surface Diffusion Modes for Pt Dimers and Trimers on Pt(100), Phys. Rev. Lett. 67, 622-625.

31. Kellogg, G. L. (1993) Diffusion of Individual Pt Atoms on Single-Crystal Surfaces of Rhodium, Phys. Rev. B 48, 11305-11312.

32. Kellogg, G. L., Wright, A. F., and Daw, M. S. (1991) Surface Diffusion and Adatom-Induced Substrate Relaxations of Pt, Pd, and Ni Atoms on Pt(001), J. Vac. Sci. Technol. A 9, 1757-1760.

33. Kellogg, G. L. (1993) The Effect of an Externally Applied Electric Field on the Reconstruction of Pt(110), Surf. Sci. 290, 295-301.

34.Schwoebel, P. R. and Kellogg, G. L. (1988) Palladium Diffusion and Cluster Nucleation on Ta(110), Phys. Rev. B 38, 5326-5331.

35. Schwoebel, P. R. and Feibelman, P. J. (1989) Extraction of Ir-Ir Adatom Interaction Strengths on $\operatorname{Ir}(100)$ from Field Ion Microscopy of Iridium Cluster Structures, Surf. Sci. 216, 263-269. 

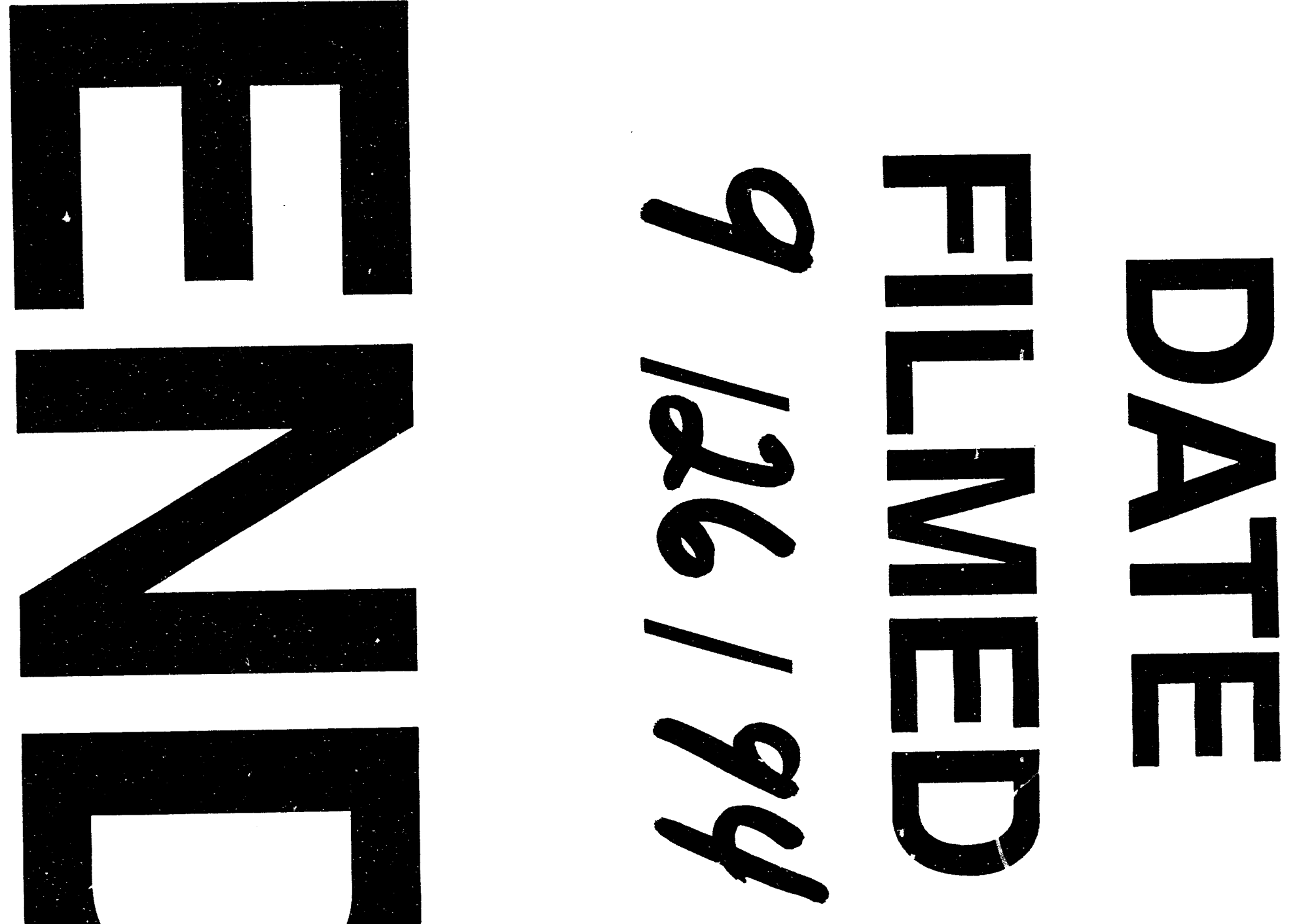
